\title{
Dislocation and Disembodiment of Interactive Body Image in Neo-Pop Art
}

\author{
Shuwen Yang ${ }^{1, *}$ \\ ${ }^{1}$ Beijing Institute of Fashion Technology, Beijing, China \\ *Corresponding author. Email: tianjiao4444@126.com
}

\begin{abstract}
With the accelerated development and popularity of digital media technology, Neo-Pop Art has expanded individuals' perceptions of the body and widened the artistic language of representing the body image. The transformation in body view stimulates the multidimensional attitude to observe the body and transcends the expression of the body in conventional aesthetic approaches. Therefore, the body image is slowly dislocated and disembodied, and a diverse artistic pattern is revealed under the convergence of technical rationality and artistic sensibility. This research concentrates on the interactive body image in Neo-Pop Art, comprising contemporary artists Yayoi Kusama and Kenny Scharf. At the same time, comparing with the expressive language of image appropriation in classic Pop Art, this paper discovers the logical clues of the transformation of the body image from being classified to being dislocated and disembodied in Neo-Pop Art. This presents the variation in how the subject is constructed in art creation and encourages the "turn" in the development of art language.
\end{abstract}

Keywords: Neo-Pop, Body image, Interactivity, Dislocation, Disembodiment.

\section{INTRODUCTION}

What is interactivity? The English etymology "interact" means the interaction, influence, and constraint between two or more individuals, which first emerged in the early 1900s in sociologist Simmel's "formal sociology" theory. It refers to that the collective activity of society should not be recognized as the objective process or behavior of individual persons but should consist of the interaction between two or more individuals, and that society as a whole develops in a series of competition, collaboration, exchange, and dispute. The term "interactivity" in Neo-Pop is also an extension of this.

In a broad sense, interaction means the activity between the artist and the object of expression or the communication between the artwork and the viewer. The audience achieves his or her reflection by observing the work of art and knows the creator's purposes. In a narrow sense, only the interaction accomplished by individuals with digital media or installation images using body movements belongs to interaction. The Neo-Pop interactive experience mainly pays attention to interactivity as the core of creation in its development. It focuses on the interaction between individuals and works; thus, it completes the process of artistic communication with the help of modern technological means and relevant equipment and instruments. Compared with public sculpture, this kind of artwork has a more varied selection of methods in the process of communication. The former realizes its value through specific materials, while the latter integrates numerous components to highlight its value. Next, in terms of formal language, there is a great difference between Pop Art and "ready-made art" in the new era. With the evolution of technology and network technology, the works have long left the conventional mode of combining images in the early Pop Art period. Instead, multi-dimensional, interactive, and playful art styles have filled the gap of diversified art creation. Then, the observer is no longer excluded from the work but is mixed into the physical space of the work, participating and communicating with it in an open forum. This form of expression provides the works themselves a sense of chance and unfinishedness and presents the audience with a Neo-Pop experience in the process of interaction. 


\section{THE INTEGRATION OF BODY AND SITUATION}

The complete individual living in the material reality does not fulfill the self-cognition of the world by "observing." In a broader sense, the individual achieves a comprehensive understanding of the world around him or her by integrating the whole body with reality. One of the purposes, why the immersive experience of Neo-Pop Art confuses the audience with reality is that it resorts to interaction over the body. As an ongoing and progressive dynamic process, on the one hand, the growth of interactive installation cannot be isolated from entertaining communication. On the other hand, it cannot be separated from the public's attention. The emphasis is also on the dynamic presence of the body image, thus effectively manifesting Mark B. N. Hansen's (1965-) vision of resorting to physical behavior in the conception of interfaces. ${ }^{1}$ The Japanese Neo-Pop artist Yayoi Kusama creates a wide range of virtual situations in several places through diverse artistic languages. Simultaneously, she continually attempts to make breakthroughs and explorations between reality and illusion, entity and space, universe and divinity. The open pattern and unexpected visual impact of Yayoi Kusama's works is one of the unique characteristics of her works. By composing visual illusions that are both real and unreal, she formulates a "heterotopia" for human beings amid pomp and circumstance, ${ }^{2}$ which is cross-border, interpenetrating, decentered or polycentric, non-

1. In his vision, Hansen assembles the specificity of human virtualization and dismantles its ontological connection to bodily emotions. Through a procedure of "analog," he connects the digital with the virtual, thus enabling the production of concrete sensations. In Hansen's viewpoint, this methodology is especially relevant in the field of virtual contexts. The immersive virtual mirroring of Neo-Pop Art creates an interactive experience projected straight onto bodily behavior through its effect on bodily perception. Mark Boris Nicola, New Philosophy for New Media, Cambridge: The MIT Press, 2004. p. 161.

2. It is first to describe the concept of "heterotopia." In 1967, the French philosopher Foucault presented a lecture on Des Espaces Autres at the French Society for the Study of Architecture. In his lecture, he first introduced the concept of "heterotopia" and explained it by the mirror reflection. He argued that when looking in the mirror, the subject sees himself or herself in a space that does not exist and that this is the mirror "utopia." Nonetheless, the mirror itself is extremely existence. When the subject in the mirror and the subject in the real world look at each other, the subject's gaze will return from the virtual space and reconstruct itself where the subject is, hence making the "heterotopia" of the mirror. Same as the mirror itself has the properties of "utopia" and "heterotopia," the positive and the negative, the internal and the external, are also interdependent, and they can invade each other and be the premise of each other. linear and shifting, etc. For instance, in Fireflies on the Water (2002), the artist emphasizes the priority and uniqueness of individual experiences by highlighting the non-prescriptive individuality of individual replies so that each individual can perceive his or her unique self in work. Yayoi Kusama is best known for mixing lighting components with mirroring elements and converting them into visually spectacular but technically minimalist installations. From complexity to simplicity, through refraction, reflection, mirroring, and neon, the audience's visual perception is constantly impacted, leading them to challenge reality in the heterogeneous space it constructs, thus provoking thoughts.

Although the entire work combines components of numerous media, the primary medium is the human being itself. ${ }^{3}$ Once the observer steps into the installation area, the artist invites them to make a secondary creation, viewing an infinite number of stars via the reflection of a mirror. Numerous beams of jewel-toned lights hang from the mirrored ceiling, linked to an automatic timing mechanism. As soon as the mirror doors close behind the observer, the room's low-tech analog transforms the entire space into a full digital video experience. ${ }^{4}$ In the illusion formed by the Pop artist, the closer the observer attempts to get, the more the viewer wants to get closer, and it will only backfire, because the illusion itself is a paradox. Every participant seems remarkably tiny in the reflection of infinite space because their individuality is weakened. In the meantime, they become aware of their common humanity as they are slowly swallowed up by the light and can look at themselves more directly. Yayoi Kusama's work is usually reminiscent of Baudrillard's theory of Simulation. By contrast, in

3. Due to the extraordinary popularity of what has been heralded as a signature 'immersive' work by the artist, visits are limited to forty-five seconds. Though brief, it is enough time to experience Kusama's ability to transform ordinary materials into a spectacular effect. Viewers enter the approximately nine-bythirteen-foot space crafted from wood, rubber, mirrors, and offthe-shelf LED lights one at a time to stand on a modest proscenium set over a shallow reflecting pool made using simple black vinyl flooring filled with a few inches of water. Mika Yoshitake, Yayoi Kusama: Infinity Mirrors, Munich: Prestel Publishing, 2017. p. 140.

4. Darkened and dampened (in both sound and humidity), the space is dimly lit by dangling strands of jeweltoned lights suspended from the mirrored ceiling. Linked to an automated timing mechanism, the lights shift between a soft pulsating glow and a stereoscopic staccato effect that becomes allencompassing. Once the mirrored door closes behind the visitor, the rooms low-tech analogue features turn the space into a complete digital imaging experience. Mika Yoshitake, Yayoi Kusama: Infinity Mirrors, Munich: Prestel Publishing, 2017. p. 140 . 
the context of immersive virtual works, "simulacrum" is not the same as "simulation." Rather, it is an expectation for the audience to ask and discover the value of reality in the state of being. As a result, from this prospect, Yayoi Kusama's works carry out the construction of "antisimulation" features. Through the implementation of multi-media expression, the boundaries of public perception are consistently breached. In such an art space, the observers are both the subject and the active subject of the work, and what this great art does is hold up a mirror that allows observers to see who they are. ${ }^{5}$ The work is a carefully choreographed interactive experience by the artist, which on the one hand, seeks a populist and immersive interactive communication. On the other hand, it also applies artistic reconstruction language to describe the fusion relationship between the environment and the body is greatly automated and digital development.

\section{THE APPEAL OF PLAYFUL FREE EXPERIENCE}

If the Sistine Chapel Ceiling (1508-1512) brought the viewer a sense of solemnity and majesty, and the works of Corita Kent (1918-1986) led crisis and trauma, then the innate playfulness of Neo-Pop Art is bound to make a visual stimulation and pleasure to the observer in the new epoch of globalization. As Bernard Bosanquet (1848-1923) declared, the initial purpose of the public's choice of play is to release their repressed emotions because "when the excess of vitality turns into activity, the animal has to play." Meanwhile, a more advanced game will be composed when the public experiences pleasure, relaxation, and joy in the viewing process.[1] This more advanced play can be explained as another kind of expression of artistic creation. Although from the prospect of several modern aestheticians, the language style of the game is not serious enough, and its playfulness is just a weak expression of the language of entertainment. Nevertheless, if we look at it from another prospect, we can notice that even the high level of play and this low level of expression are similar in sensory stimulation and pleasure. In the

5. This is not a metaphor or analogy but an actualization of the cinematic concept in which a subject is replicated and often reconfgured in smaller forms. Inside Kusama's Infinity Mirrored Room-The Souls of Millions of Light Years Away, the viewer observes the multiplication of his or her specifically, most visitors endure the hours-long wait forown image from all geometrically conceivable angles. Mika Yoshitake, Yayoi Kusama: Infinity Mirrors, Munich: Prestel Publishing, 2017, p. 140 . playing procedure, the beauty of the body approaches its maxium, and it is infiltrated with harmony and rhythm in the development process.

In his work Letters Upon the Aesthetic Education of Man (1794), Friedrich Schiller (17591805) proposed that the impulse and inspiration for artistic piece originate from an emotion named the "spielterieb." This "spielterieb" is set up by the "instinct for form" and the "sensuous instinct." If the "instinct for form" is manifested in multimedia installations in the process of globalized art development, and art is evolved rationally in science and technology. In that case, the "sensuous instinct" is reflected in the aesthetic pleasure of play, presenting the audience perform the impulse of desire in the immersive interactive space.[2] This playful pleasure is a powerful method to free the viewer from reality, and it is a happiness that the subject creates from the experience of a free state. This is the same as the viewer encounters aesthetic pleasure; the state of freedom carried about by the rhythm and harmony of the body can also provide the observer happiness in the aesthetic process of play. This playful language of creation is evident throughout Kenny Scharf's (1958-) work, who is an American Neo-Pop artist. In his exhibition called Cosmic Cavern (2012), the immersive sensory experience immerses the observer fully in a fluorescent cosmic world. At the moment, it is surrounded by graffiti tags, debris, and daily objects reimagined as cartoon characters. By adopting huge areas of vibrant fluorescent colors presents the artist's attempt to return to this extremely artificial language of colorful expression, as critics have stated of his creation, "building a cheerful smorgasbord of atmosphere in its gaudy colors."[3] This surreal world comes to life under the dazzling fluorescent lights, reminiscent of American clubs or discos during the 1980s. The Cosmic Cavern manifests an integration of Scharf's Pop Art language and surrealism, mixing cartoonish imagery, consumer symbols, graffiti, dreamy futuristic environments, and bright, dazzling color changes. Correspondingly, it presents a combination of Scharf language and surrealist style. ${ }^{6}$ In the immersive and interactive expression

6. Scharf desires to "transcend the elite of art and combine with popular culture. He chooses his material from comics because he mentions that such imagery has turned into part of the public's collective unconscious. Scharf's earliest drawings were based on the Hanna-Barbera characters he loved as a child. However, until 1983, Scharf started designing his cartoon characters, which he considered "the offspring of surrealist fantasy. Bradford R. Collins, Pop art: the independent group to Neo Pop, 1952-90, New York: Phaidon, 2012. p. 400. 
is full of the spirit of the game and the sense of unique surprise. Its playful, relaxing, pleasant, and entertaining style attracts the viewer to engage in the game personally and composes a new style of aesthetic connection in the process. Consistently, the body no longer needs to bear too much repression and heavy implications but only requires perception and interpreting its behavior with its engagement. $^{7}$

\section{DISLOCATION AND DISEMBODIMENT OF THE BODY}

In virtual art's immersive interactive experience creations, the body image produces an aesthetic feature of "dislocation" and "disembodiment." As a constituent component in the virtual illusion space, the body has been fully detached from the subject's physicality, and the material properties within the initial physiological dimension have been increasingly dissolved. The process of being modified and renovated shows a body image that is very distinctive from the creation of classic Pop Art. This is intimately associated with the postmodernist trend and the theory of acceptance of aesthetics. ${ }^{8}$ Postmodernism promotes the concept of rebellion against authority and fluidity, pluralism, decentering, and uncertainty. In addition, its acceptance aesthetics highlights the range of ideas of initiative, freedom, and creativity, all of which have had a meaningful influence on the development of Pop Art in the new era. In the period when classic Pop Art was popular, the identity of the artist's subject was clear, while the body image in work was flawless and direct. The older Pop artists executed an aesthetic appreciation

7. The desire to integrate high art with low art is also evident in the fun of the Pollockian linear entanglement performed within the work. Although most people unanimously assume that the function of Scharf's work is to allow himself and the audience to return to the happiness and beauty that they encountered in childhood in front of the color TV screen. However, one critic has suggested that this impulse is rooted in Scharf's disillusionment and what his generation felt at the discovery. They thought the world had not changed for the better and that they had recognized this since childhood. Bradford R. Collins, Pop art: the independent group to Neo Pop, 1952-90, New York: Phaidon, 2012. p. 400.

8. Acceptance aesthetics, or acceptance theory, was founded in the mid-1960s by Hans Robert Jauss (1921-1997) in Germany. It principally mentions that the work's entertainment and educational function should be displayed in the reading process, and the display is the procedure of the final completion and vitality of the work. The audience is active and free in this procedure and is the driving force behind the development of artistic creation. Consequently, the work's reception is governed by the work itself and the audience. Bradford R. Collins, Pop art: the independent group to Neo Pop, 1952-90, New York: Phaidon, 2012. p. 400. of their artistic conceptions through symbolic and schematic body images, which turned into an externalized expression of their aesthetic perception. The artist's control over the image occupied an extremely central position and imposed the form and meaning of the creation on the observer in the dissemination process. In this procedure, the audience was passively explaining the symbolic intent sent by the creation. However, in the post-1980s Neo-Pop Art, the multi-media and interdisciplinary language of art caused the artists to not now as formerly confine themselves to traditional art. Alternatively, they emerged as a multi-disciplinary group, in which workers from separate disciplines and domains of the study were each making their contribution to the work. By constantly decomposing and combining, this creation mode also made the body image in the works more variable, blurred, and even faded.

Whether transformation will occur in the future, the network system organized by the individual body and the external world, the interactive behaviors accomplished by the body in the virtual space, etc., will prompt the audience to reconsider the human body? What is the identity of a human being? Furthermore, what is human nature? Accordingly, with the growth of science and technology as well as genetic and biological technology, the individual will no more be restricted to how to manifest himself or herself in such a variable space and present his or her value. Rather, it is more about what variety of body image we actually want to realize when the body enrolls in the image world.[4] Moreover, the sort of immersive interactive experience allows such creations to take on a dynamic, open-ended situation. It also demands that the passive audience in traditional artworks be transformed into an active participant who is free to be involved in creating the work. In such a way, the body image in traditional artworks is converted from the comprehensive and definite symbolic body image in classic Pop Art to a multifaceted and unpredictable body image. Subsequently, this unpredictability reduces the artist's control over the work and the "dislocation" and "disembodiment" of the certain body image in work. The Neo-Pop interactive works form a "believable" world for the public, and the observers may determine to enter with a critical attitude or a judgmental mind. Notwithstanding, most of them are still curious and entertaining to experience the illusionary scenes that alternate with and parallel the real world. Under the dual effect of dislocation and 
disembodiment, the observer realizes the experience of "being there," and the illusion of imitation overwhelms the body in space and continuously consumes it.

\section{CONCLUSION}

The evolution of technology and the development of communication surely cause a change in the public's conception of the body. The freedom, openness, and post-modernity in the context of globalization improve the expression of body image in the new generation of Pop Art. Thus, it prolongs the visual aesthetic dimension of the public in the unending reciprocation of breaking and rebuilding. The transformation of the view of the body stimulates a multi-dimensional approach to inspecting the body and transcends the traditional aesthetic expression of the body image. From its cultural character and expressive potential, the body enriches and creates a new mode of artistic language. Whether virtual body image, multidimensional body image, or interactive body image, they all turn into a new language in the Neo-Pop Art mode, causing the body image to present a diversified pattern in this era. In addition, their creation also manifests the newness of Neo-Pop Art. The coverage of the Internet and technology allowed Neo-Pop Art to evolve from an extension of the visual senses to an expansion of the public's emotional and intellectual sphere, thus leading the audience to break free from the ritualization of conventional ideologies. Transfer from visual aesthetics to conceptual aesthetics, spiritual aesthetics, and thinking aesthetics; From a spiritual status of collective identity to a visual activity combined with various meanings, for instance, selfemotion, self-desire, self-entertainment, recreation, and exploitation, this is a resonance in the inner logic and deep emotion of the body image that comes from the extension of visual experience. Additionally, in the operation of the globalized art mechanism, the dissemination of art information converted the core to the development of art at this step. "Information dissemination" has approved artworks to achieve unprecedented popularity and reputation. On the one hand, the commercial value of art has been advanced. On the other hand, the propaganda of the artworks via the communication media also presented an increasingly prominent social effect, which led to the transfer of the interactive body image in Neo-Pop Art in the procedure of gradual dislocation and disembodiment.

\section{AUTHORS' CONTRIBUTIONS}

This paper is independently completed by Shuwen Yang.

\section{REFERENCES}

[1] [English] Bernard Bosanquet, translated by Zhang Jin. History of Aesthetics. Guangxi Normal University Press. 2009. p. 257.

[2] [English] Friedrich Schiller, translated by Zhang Yuneng. Letters Upon the Aesthetic Education of Man. Nanjing Yilin Publishing House. 2009 edition.

[3] Wang Hongyi. Western Contemporary Art: Counter-artistic art history. Harbin Institute of Technology Press. 2008 edition. p. 196.

[4] [German] Sybille Kraemer, translated by Sun Heping. Medium, Messenger, Transmission: An Approach to Media Philosophy. China Social Science Press. 2008 edition. pp. 125, 126, 138.

[5] [American] Lucy R. Lippard, Lawrence Alloway, Nancy Marmer, et al. Pop art, London: Thames and Hudson 1970.

[6] [English] Lawrence Alloway, American pop art, New York: Collier Books, 1974a.

[7] [American] Bradford R. Collins, Pop art: the independent group to Neo Pop, 1952-90, New York: Phaidon, 2012.

[8] [English] Marco Livingstone, Pop art: a continuing history, London: Thames and Hudson.

[9] [American] Mark B. N. Hansen, 2004. New Philosophy for New Media [M]. Cambridge: The MIT Press. 